PMo-26 / ISEC2003

Submitted on 12 July 2003

\title{
Design and Implementation of Circuit Components of the SFQ Microprocessor,
} CORE1

N. Nakajima ${ }^{1)}$, F. Matsuzaki ${ }^{1)}$, Y. Yamanashi ${ }^{1)}$, N. Yoshikawa ${ }^{1)}$, M. Tanaka ${ }^{2)}$, T. Kondo $^{2)}$, A. Fujimaki ${ }^{2)}$, H. Terai $^{3)}$ and S. Yorozu ${ }^{4)}$.

1) Department of Electrical and Computer Engineering, Yokohama National University, 79-5 Tokiwadai, Hodogaya-ku, Yokohama 240-8501, Japan

2) Department of Quantum Engineering, Nagoya University, Furo-cho, Chikusa-ku, Nagoya 464-8603, Japan

3) Communication Research Laboratory, 588-2 Iwaoka, Nishi-ku, Kobe 651-2492, Japan

4) SRL-ISTEC, 34 Miyukigaoka, Tsukuba 305-8501, Japan

*Corresponding author.

Prof. Nobuyuki Yoshikawa

Postal address: Department of Electrical and Computer Engineering, Yokohama National University, Tokiwadai 79-5, Hodogaya, Yokohama 240-8501, Japan

Phone: +81-45-339-4259

Fax: $+81-45-338-1157$

E-mail: yoshi@yoshilab.dnj.ynu.ac.jp 
Short title: Design and Implementation of Circuit Components of the SFQ Microprocessor, CORE1

PACS code: $85.25 . \mathrm{Hv}, 85.40 . \mathrm{Bh}$

\begin{abstract}
We have designed, implemented and tested several circuit components of a prototype of the SFQ microprocessor, CORE1. The CORE1 is a synchronously clocked 8-bit microprocessor based on a bit-serial architecture. We have designed its circuit components using a cell-based design approach and an automated top-down CAD environment. We have successfully obtained correct operations of all circuit components, including an instruction register, a program counter and a controller.
\end{abstract}




\section{Introduction}

One of the most attractive applications of SFQ digital circuits [1] is high-end computing application $[2,3]$, where advantages of SFQ circuits in terms of power and speed can be fully utilized. We have been developing a small SFQ microprocessor, which is called CORE1, in order to demonstrate feasibility of large-scale SFQ digital circuits [4]. A block diagram of the CORE1 is shown in Fig. 1, which is a synchronously clocked 8-bit microprocessor based on a bit serial-architecture. The CORE1 has seven instructions and a 32-byte address space. Its micro-architecture is almost similar to that of our previous asynchronous SFQ microprocessor [5] except that in the CORE1 all circuit components are clocked synchronously by a single clock generator placed in a controller. The bit-serial architecture is employed to increase the clock frequency into the utmost limit of the SFQ circuit. Its target clock frequency is 16 $\mathrm{GHz}$, which is limited by the operation speed of an ALU with a carry feedback loop. We have designed and implemented all circuit components of the CORE1 using the NEC $\mathrm{Nb} 2.5 \mathrm{kA} / \mathrm{cm}^{2}$ standard process.

In this paper, we will show details of circuit designs and test results of several circuit components of the CORE1, that is, an instruction register (IR), a program counter (PC) and a controller.

\section{Design and Test of CORE1 Circuit Components}

We have designed all circuit components of the microprocessor using the CONNECT cell library [6], which is consists of more than two hundreds of basic cells. Specially developed top-down CAD tools, including a logic simulator, a circuit simulator and a layout extraction tool, are also used to design the large-scale circuit 
components containing several hundreds of Josephson junctions.

\section{A. Instruction Register}

A block diagram of the IR is shown in Fig. 2. The IR fetches a bit-serial 8-bit word from a RAM at $16 \mathrm{GHz}$, converts it into a parallel 3-bit opcode and a parallel 5-bit operand, and sends them to the controller and the PC, respectively (see Fig. 1).

A schematic of the IR is shown in Fig. 3. A circuit consists of seven D flip-flop cells, eight NDRO cells and some wiring cells. A layout size is $240 \mu \mathrm{m} \times 1,680 \mu \mathrm{m}$, and the number of the Josephson junctions is 448 . The total bias current is estimated to be 57.2 $\mathrm{mA}$. Logic simulation indicates that its de bias margin ranges $-33 \% \sim+29 \%$.

Fig. 4 shows test results, where the output signals of top two bits are displayed when the input data pattern is (01111111). We have also measured the other 6-bit output signals and confirmed the correct operation of the whole circuit. The on-chip high-speed test shows that the dc bias margin of the 8 -bit IR is $-27.2 \% \sim+19.1 \%$ at $16 \mathrm{GHz}$.

\section{B. Program Counter}

The PC keeps a current 5-bit address of the program on the RAM and counts up at every system cycle. A new address is also loaded from the IR when a JUMP operation is executed. A block diagram of the PC is shown in Fig. 5. In operation, the PC holds the data at an internal register when a parallel 5-bit data are inputted. The internal counter is updated by the data in the internal register when the Load_PC_trg is inputted. The internal counter also counts up its data when Inc_PC_trg is inputted, and outputs them when the PC_trg is applied.

A schematic of the PC is shown in Fig. 6. The PC consists of five D flip-flop cells, five T flip-flop cells and five NDRO cells with a reset function. Its layout size is 
$480 \mu \mathrm{m} \times 1,000 \mu \mathrm{m}$, and the number of the Josephson junctions is 548 . The total bias current is estimated to be $64.3 \mathrm{~mA}$. Logic simulation indicates that its dc bias margin is $-32 \% \sim+29 \%$.

Fig. 7 shows low speed test results of the PC. In the test counting up and readout operations are repeated nine times, then a new input data (11111) is loaded by applying Load_PC_trg. In the figure only bottom three signals are shown. One can see that counting up and load operations are obtained correctly. We have also measured the other 2-bit signals and confirmed the correct operation of the whole circuit. The dc bias margin of the PC is found to be $-12.5 \% \sim+30.5 \%$.

\section{Controller}

The controller handles all circuit components in the microprocessor by providing thirteen kinds of trigger pulses and $16 \mathrm{GHz}$ clocks for appropriate circuit blocks. Its operation is determined by a 3-bit opcode and the microprocessor state, where the microprocessor has seven phases for every machine cycle [4]. A sequence table of the controller is shown in Table 1. In the table, squares with " 1 " correspond to an output of 1-bit SFQ trigger pulses and "12" represents an output of 12-bit SFQ clock pulses to circuit blocks in the microprocessor.

We have designed the controller using a binary decision diagram (BDD) [7]. We have employed the BDD SFQ logic in the complex controller design because a logic synthesis method is well developed in this design approach. The BDD of the controller is shown in Fig. 8. In the figure, open circles represent on-to-two binary switches, which switch input SFQ pulses into one of two outputs depending on their internal state as denoted in the figure. A schematic of the controller is shown in Fig. 9. The controller 
consists of fifteen resettable D flip-flop cells with complementary output and five NDRO cells. Its layout size is $1,560 \mu \mathrm{m} \times 1,720 \mu \mathrm{m}$, and the number of the Josephson junctions is 2,399. The total bias current is estimated to be $302 \mathrm{~mA}$. Logic simulation indicates that its de bias margin is $-30 \% \sim+29 \%$. Figure 10 is a photograph of the controller.

We have confirmed the correct operation of the controller by measuring all output signals. Its dc bias margin is summarized in Fig. 11. The de bias margin of the whole circuit is $-1.4 \% \sim+21.1 \%$, whereas the dc bias margin except a $16 \mathrm{GHz}$ clock generator is $-19.4 \% \sim+21.1 \%$. The decrease in the dc bias margin of the whole circuit containing the $16 \mathrm{GHz}$ clock generator is due to deterioration of our on-chip high-speed test system in this sample.

\section{Conclusions}

We have designed and implemented an 8-bit instruction register, a 5-bit program counter and a controller of the SFQ microprocessor CORE1 by using a cellbased design methodology. Relatively large dc bias margins ranging about $\pm 20 \%$ were obtained for each circuit component from low-speed and high-speed tests.

\section{Acknowledgements}

This work was supported by the New Energy and Industrial Technology Development Organization (NEDO) through ISTEC as Collaborative Research and Superconductors Network Device Project. 


\section{References}

[1] Likharev K K and Semenov V K 1991 RSFQ Logic/Memory Family: A New Josephson-Junction Technology for Sub-Terahertz-Clock-Frequency Digital Systems IEEE Trans. Appl. Supercond. 1 3-28

[2] 9. Dorojevets M, Bunyk P and Zinoviev D 2001 FLUX Chip: Design of a 20-GHz 16-bit Ultrapipelined RSFQ Processor Prototype Based on 1.75- $\mu \mathrm{m}$ LTS Technology IEEE Trans. Appl. Supercond. 11 326-332

[3] Fujimaki A, Takai Y and Yoshikawa N 2002 High-End Server Based on Complexity-Reduced Architecture for Superconductor Technology IEICE Trans. Electron. E85-C 612-616

[4] Tanaka M, Matsuzaki F, Kondo T, Nakajima N, Yamanashi Y, Terai H, Yorozu S, Yoshikawa N, Fujimaki A and Hayakawa H 2003 Component test toward single-fluxquantum processors to be published in PhysicaC.

[5] Yoshikawa N, Matsuzaki F, Nakajima N, Fujiwara K, Yoda K and Kawasaki K 2003 Design and Component Test of a Tiny Processor Based on the SFQ Technology IEEE Trans. Appl. Supercond. 13 441-445

[6] Yorozu S, Kameda Y, Terai H, Fujimaki A, Yamada H and Tahara S 2002 A single flux quantum standard logic cell library PhysicaC 378-381 1471-1474

[7] Yoshikawa N and Koshiyama J 2001 Top-Down RSFQ Logic Design Based on a Binary Decision Diagram IEEE Trans. Appl. Supercond. 11 1098-1101 


\section{Figure Captions}

Fig. 1. A block diagram of the SFQ microprocessor, CORE1.

Fig. 2. A block diagram of the 8-bit instruction register.

Fig. 3. A circuit schematic of the 8-bit instruction register.

Fig. 4. A test result of the 8-bit instruction register..

Fig. 5. A block diagram of the 5-bit program counter.

Fig. 6. A circuit schematic of the 5-bit program counter.

Fig. 7. A test result of the 5-bit program counter. (a) Input data pattern and (b) output signals.

Fig. 8. Binary Decision Diagram of the controller.

Fig. 9. A circuit schematic of the controller.

Fig. 10. A chip photograph of the controller.

Fig. 11. Tested dc bias margins of the controller. 


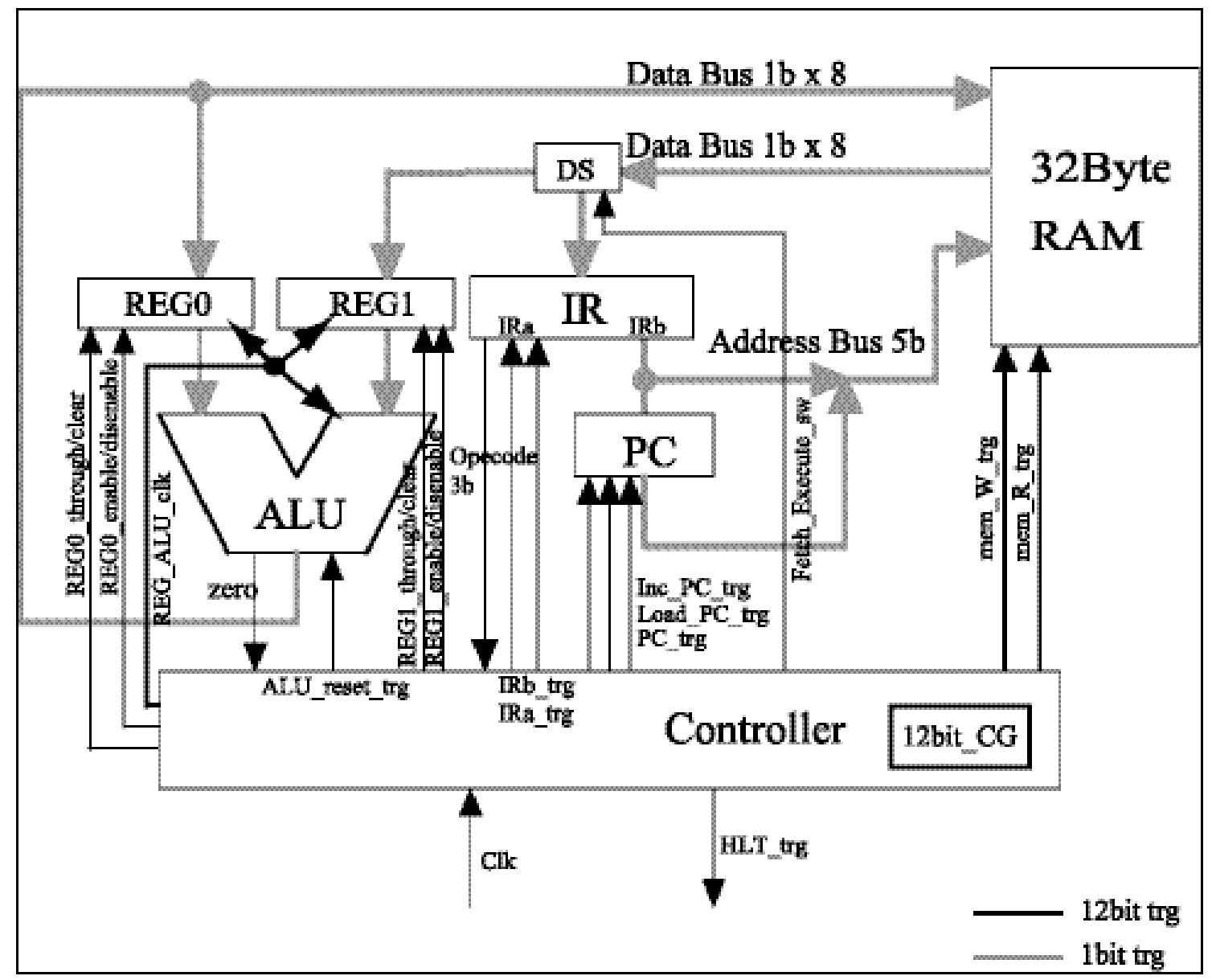

Figure 1 


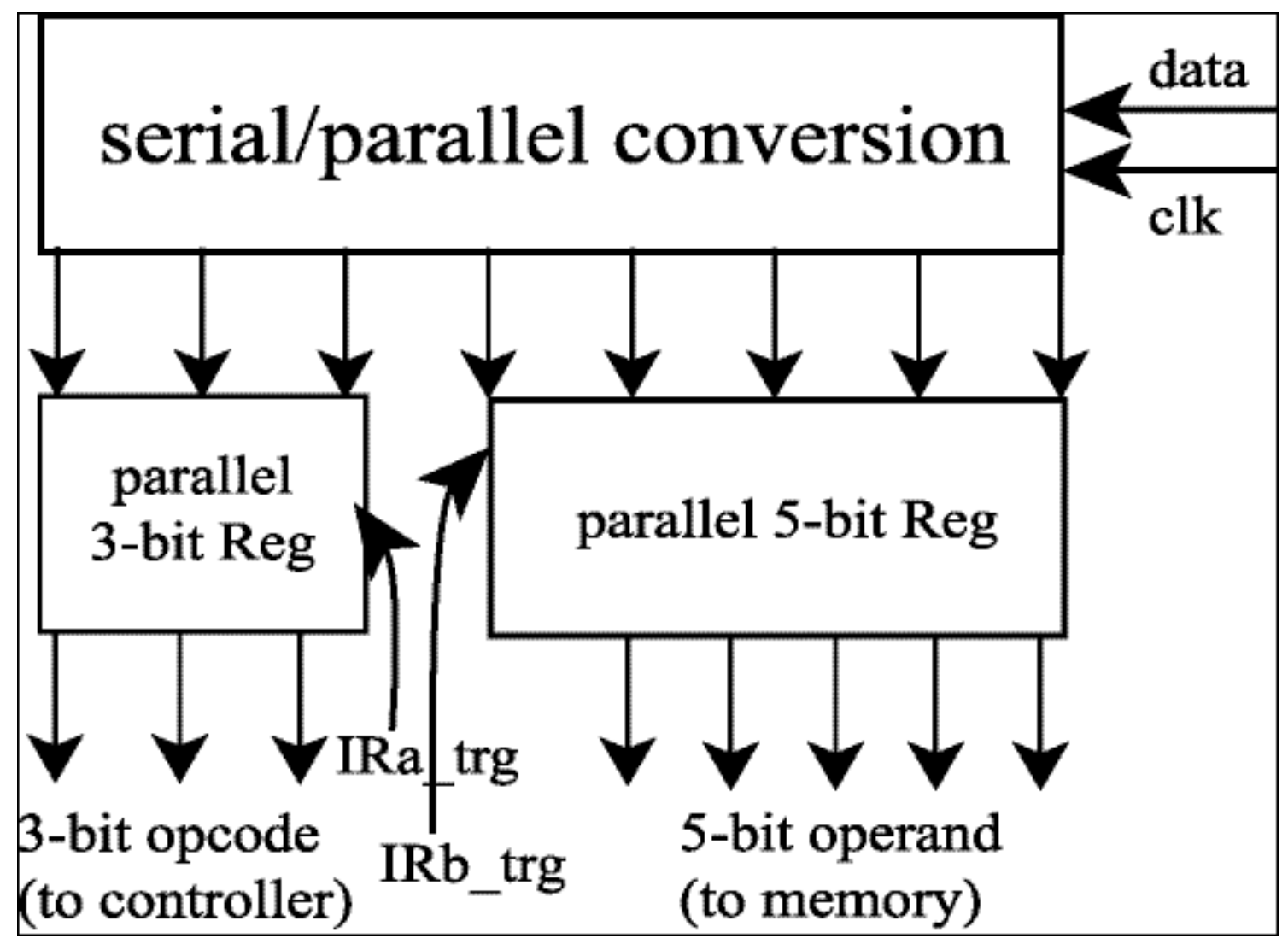

Figure 2 


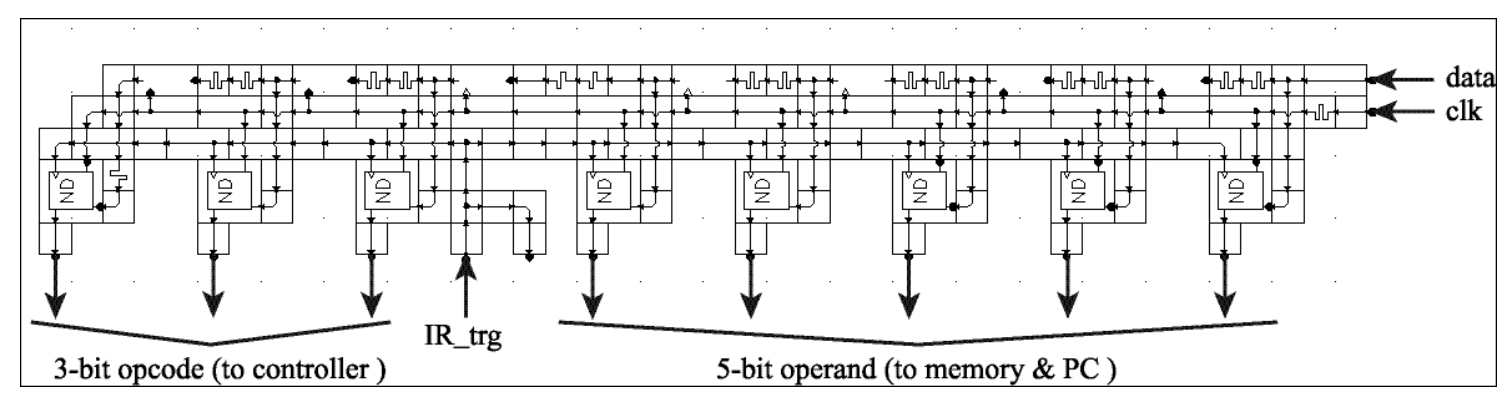

Figure 3 


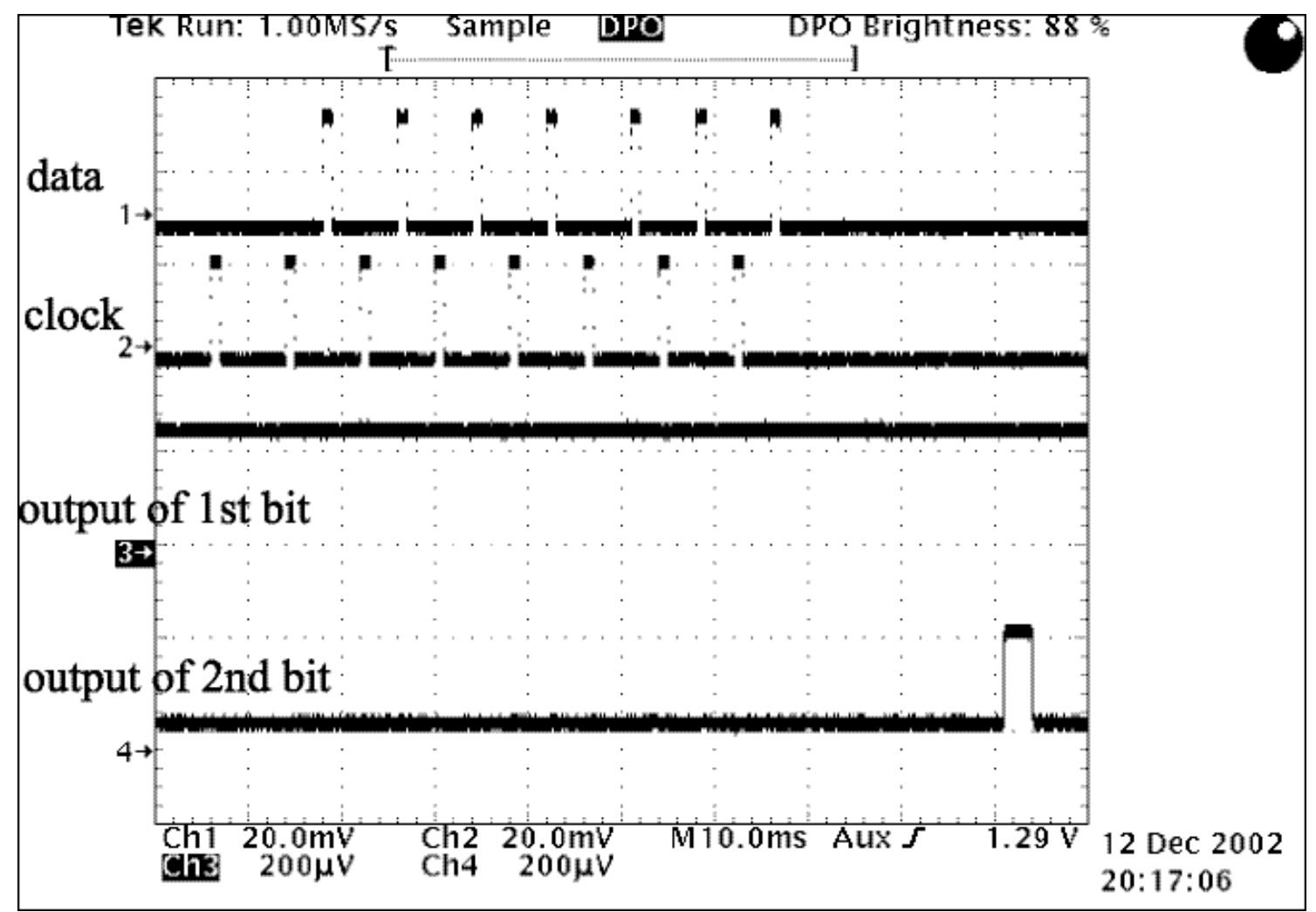

Figure 4 


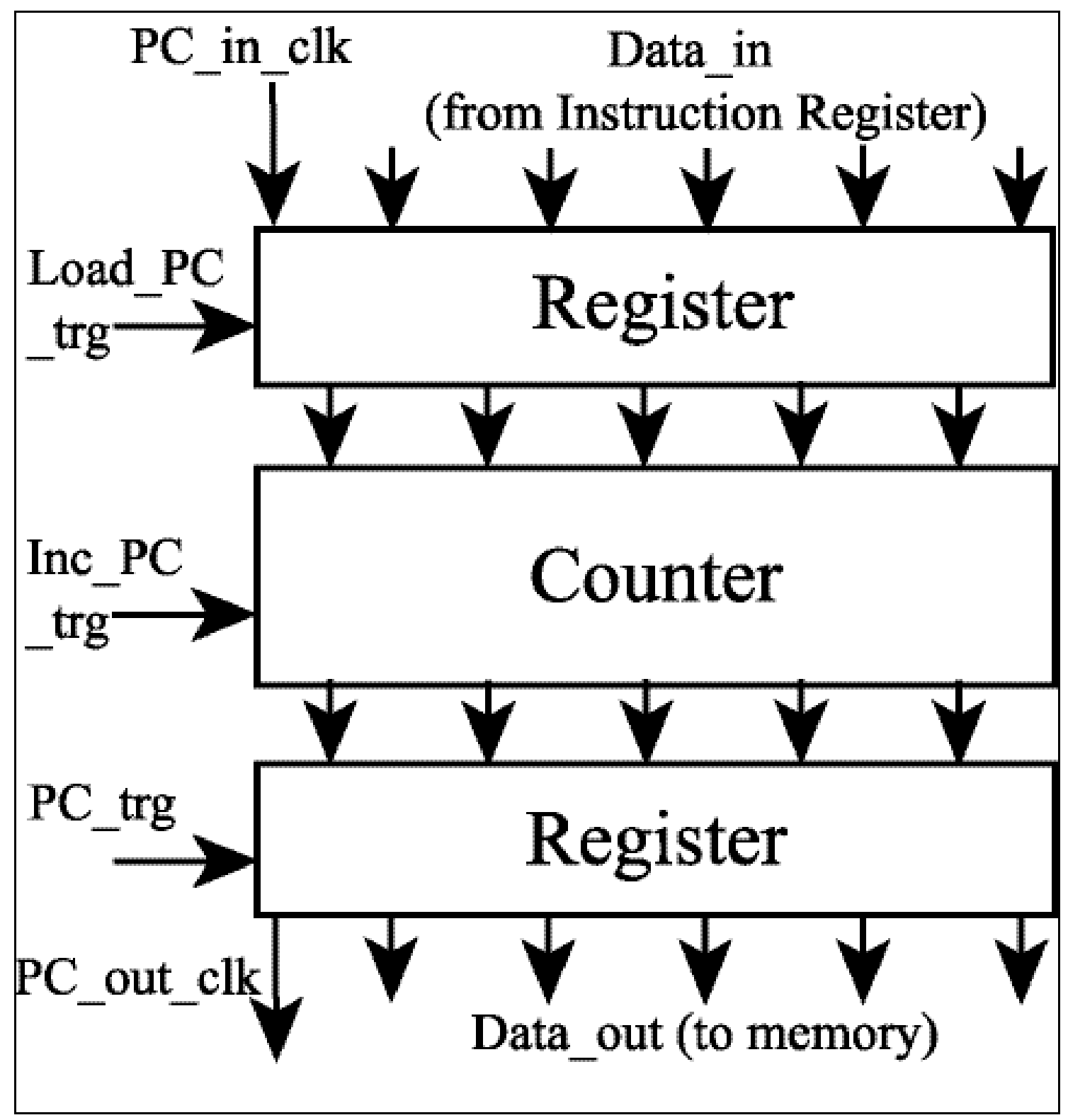

Figure 5 


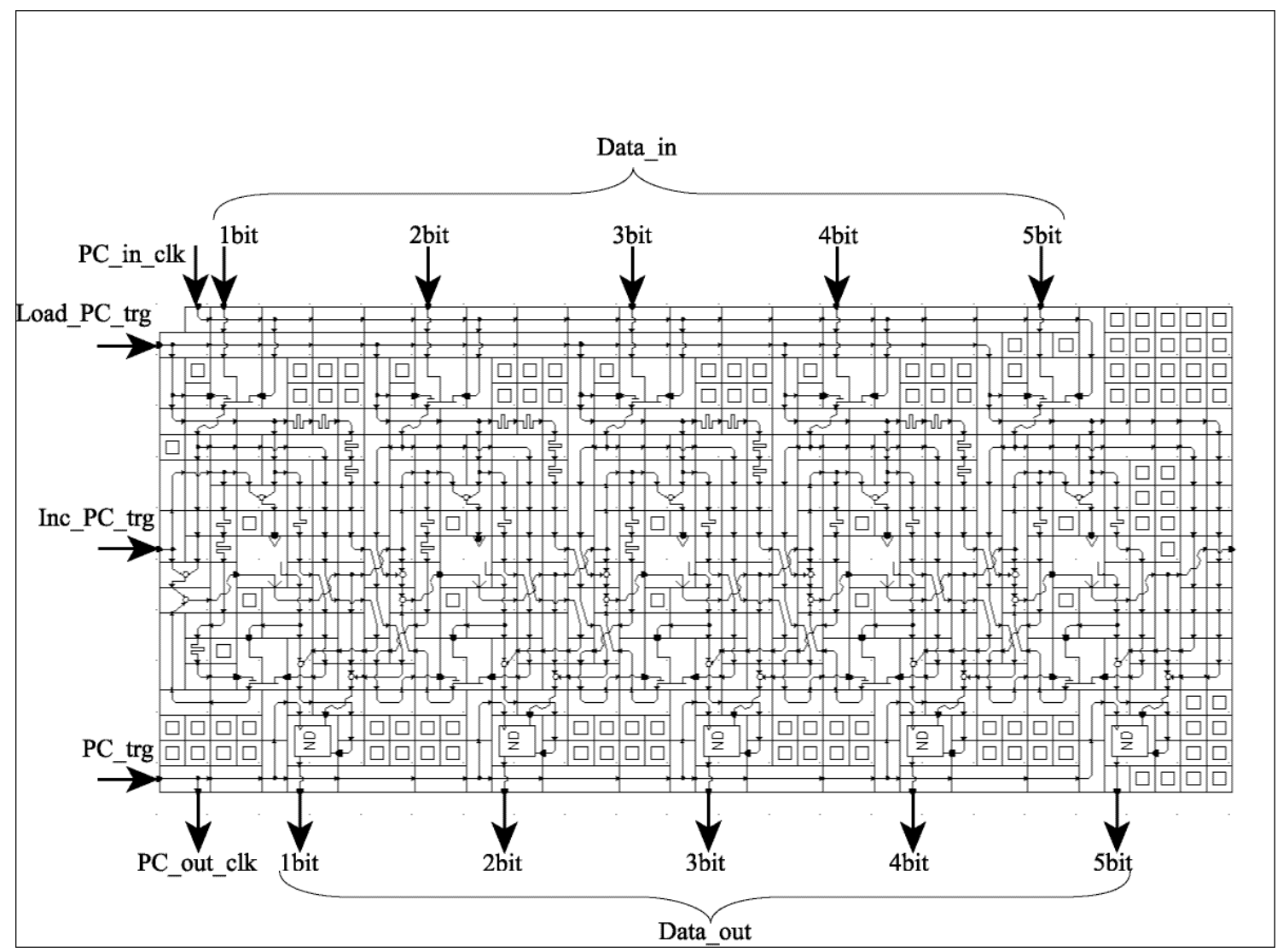

Figure 6 


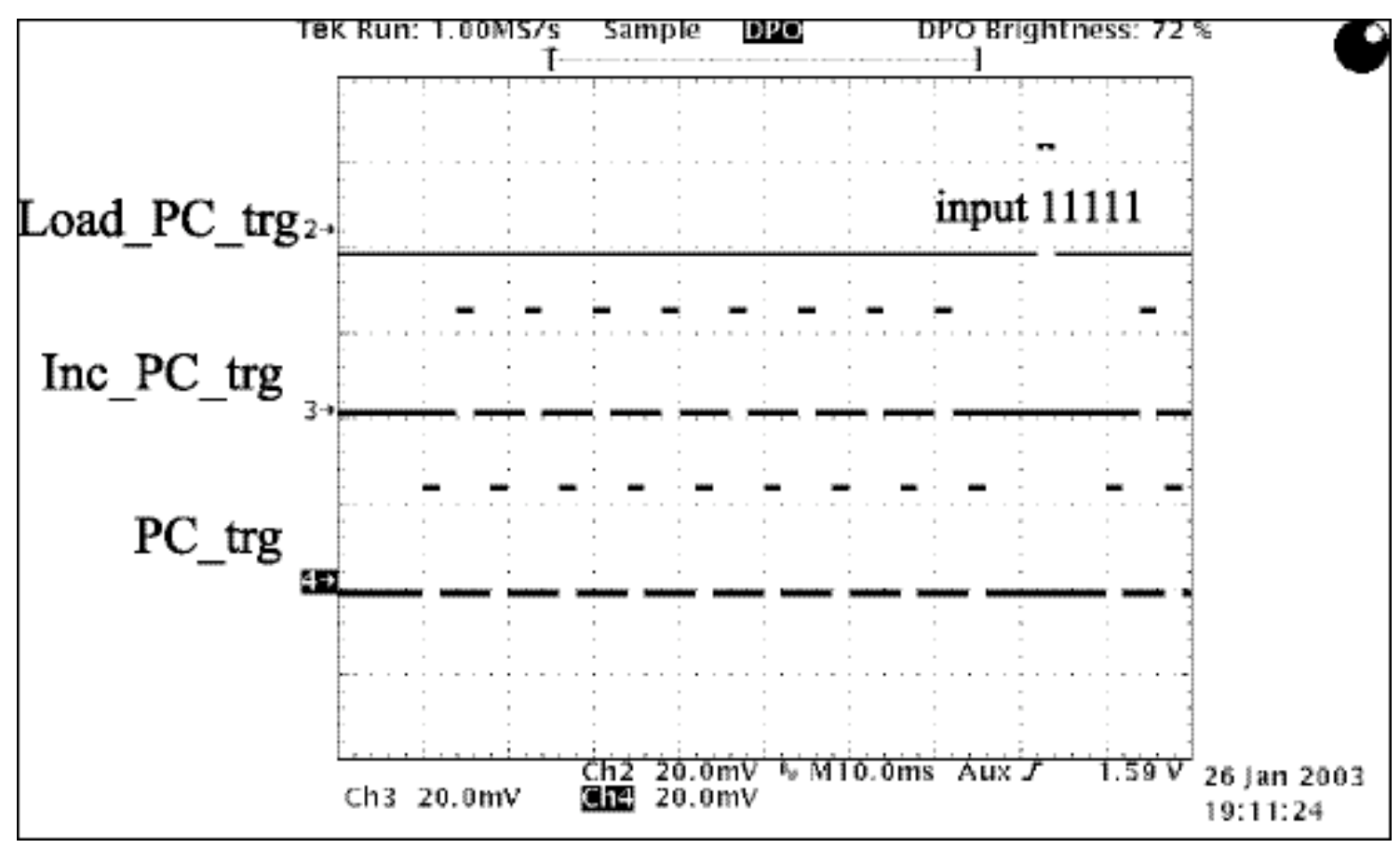

(a)

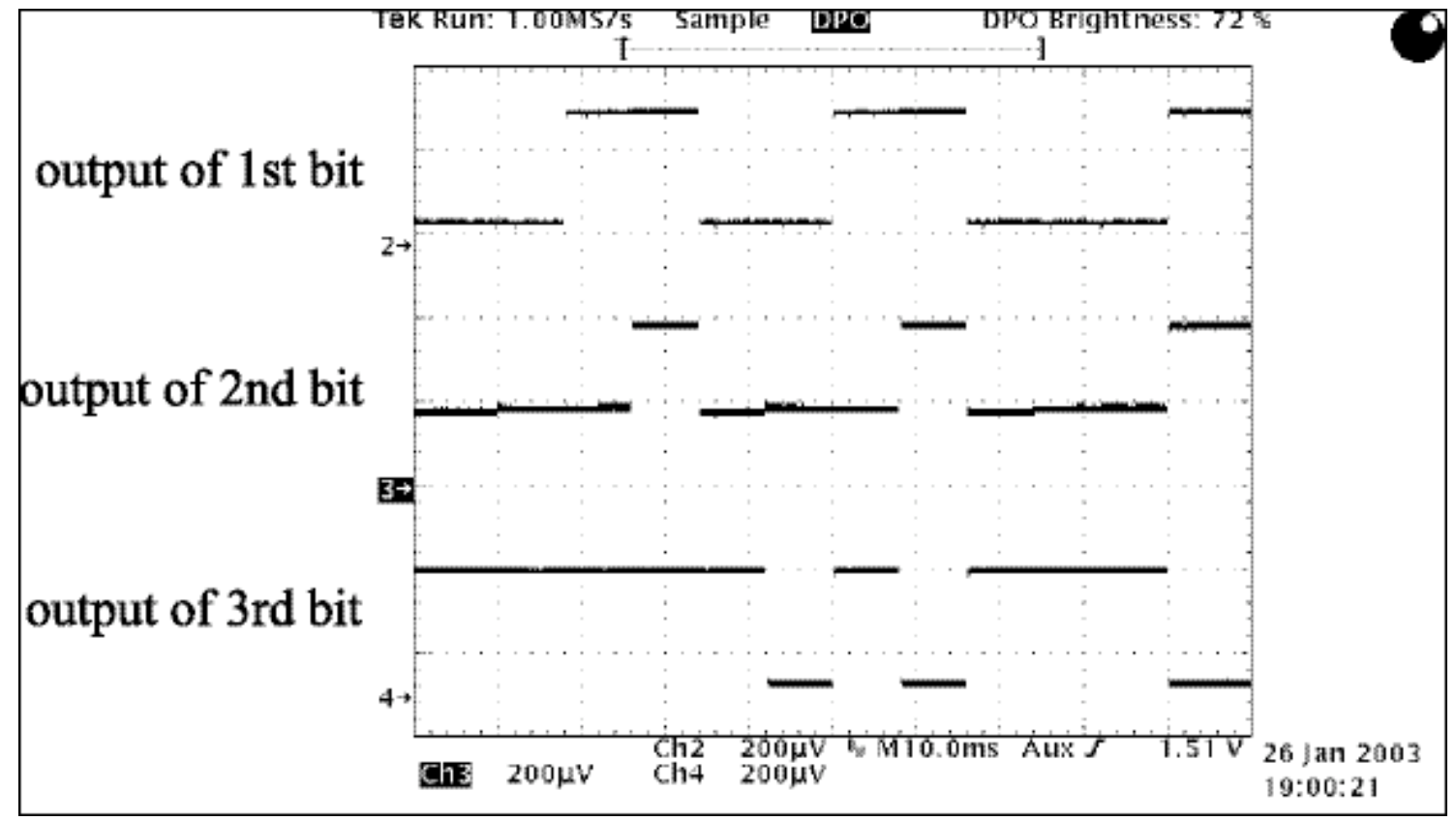

(b)

Figure 7 


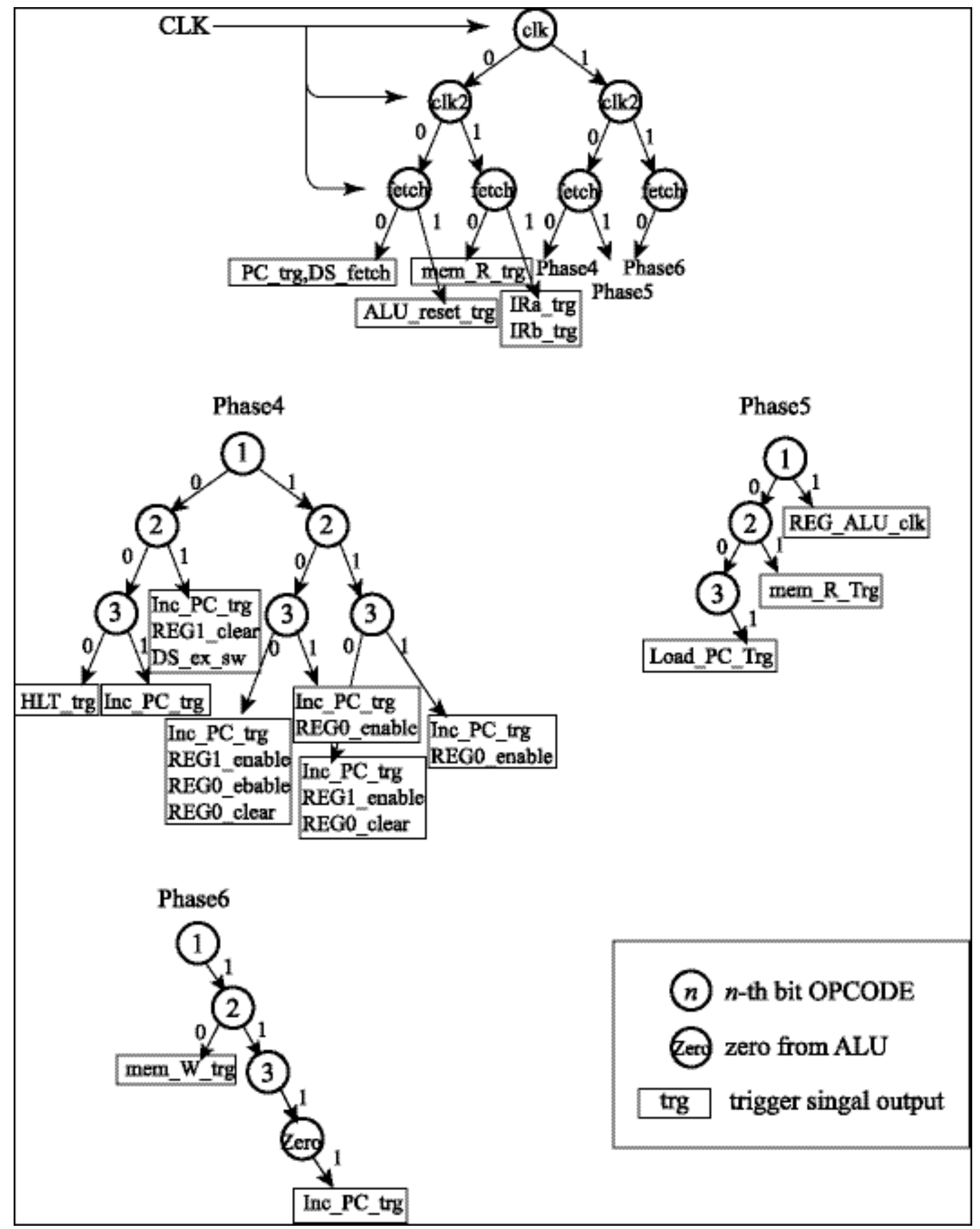

Figure 8 


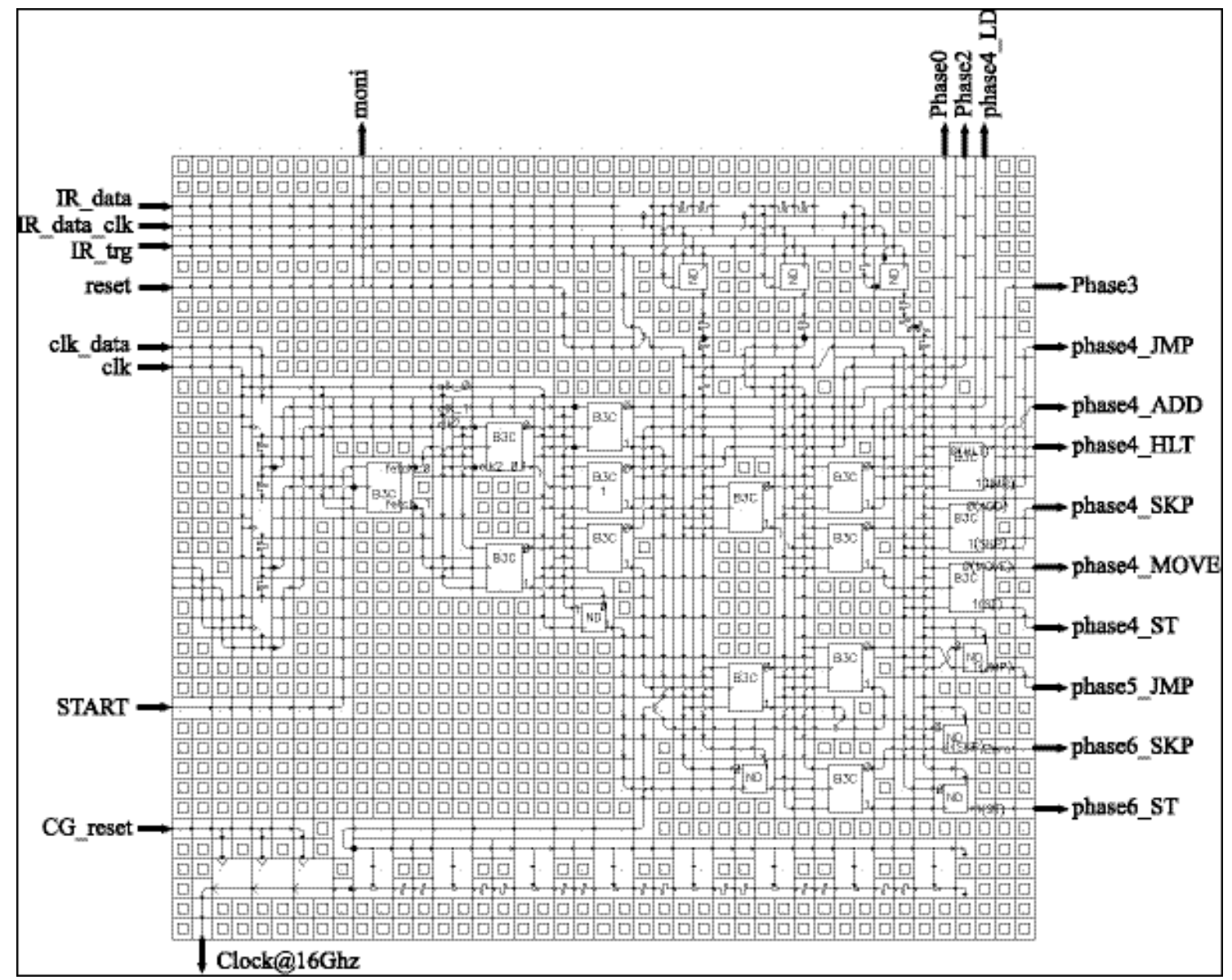

Figure 9 


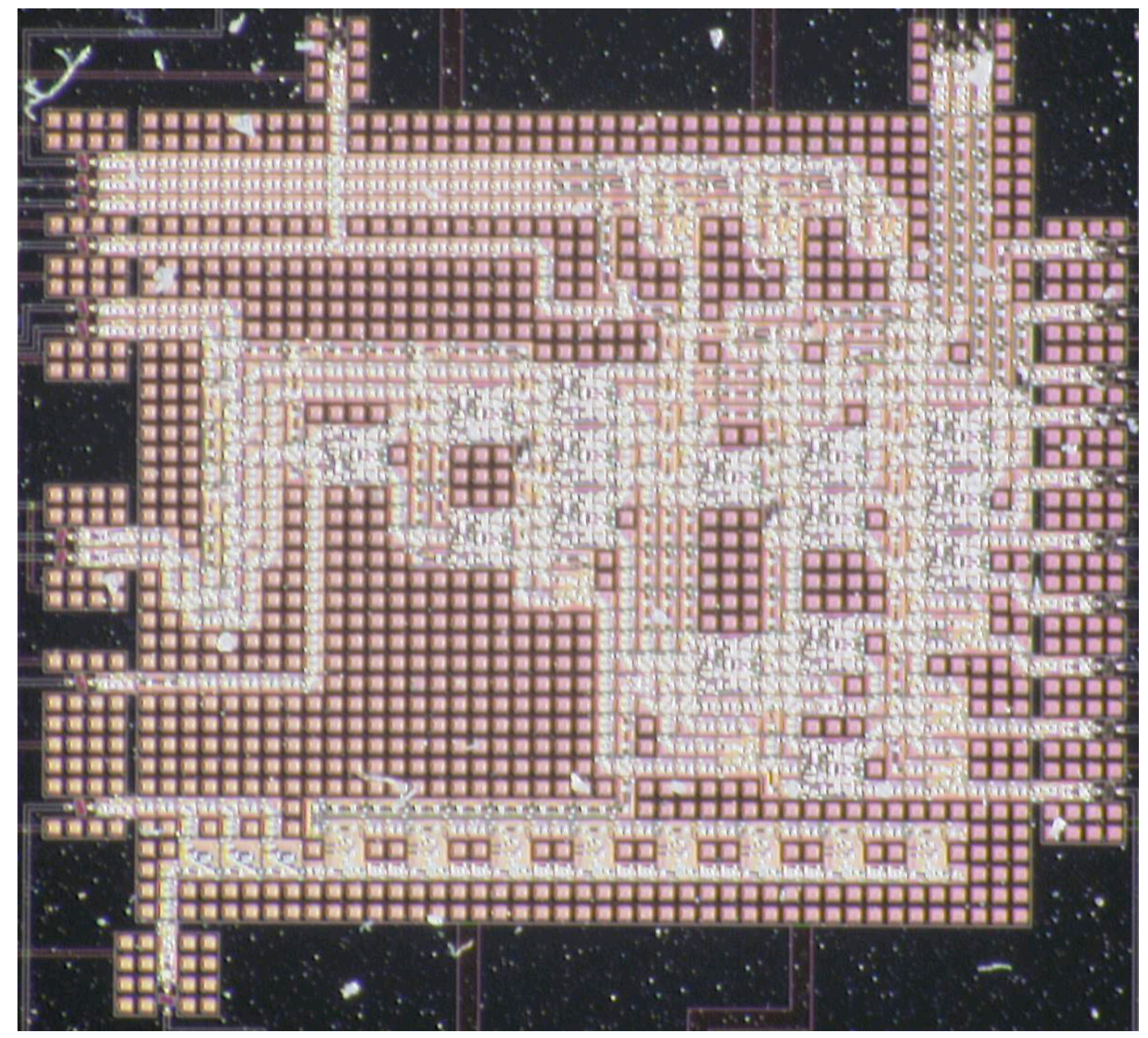

Figure 10 


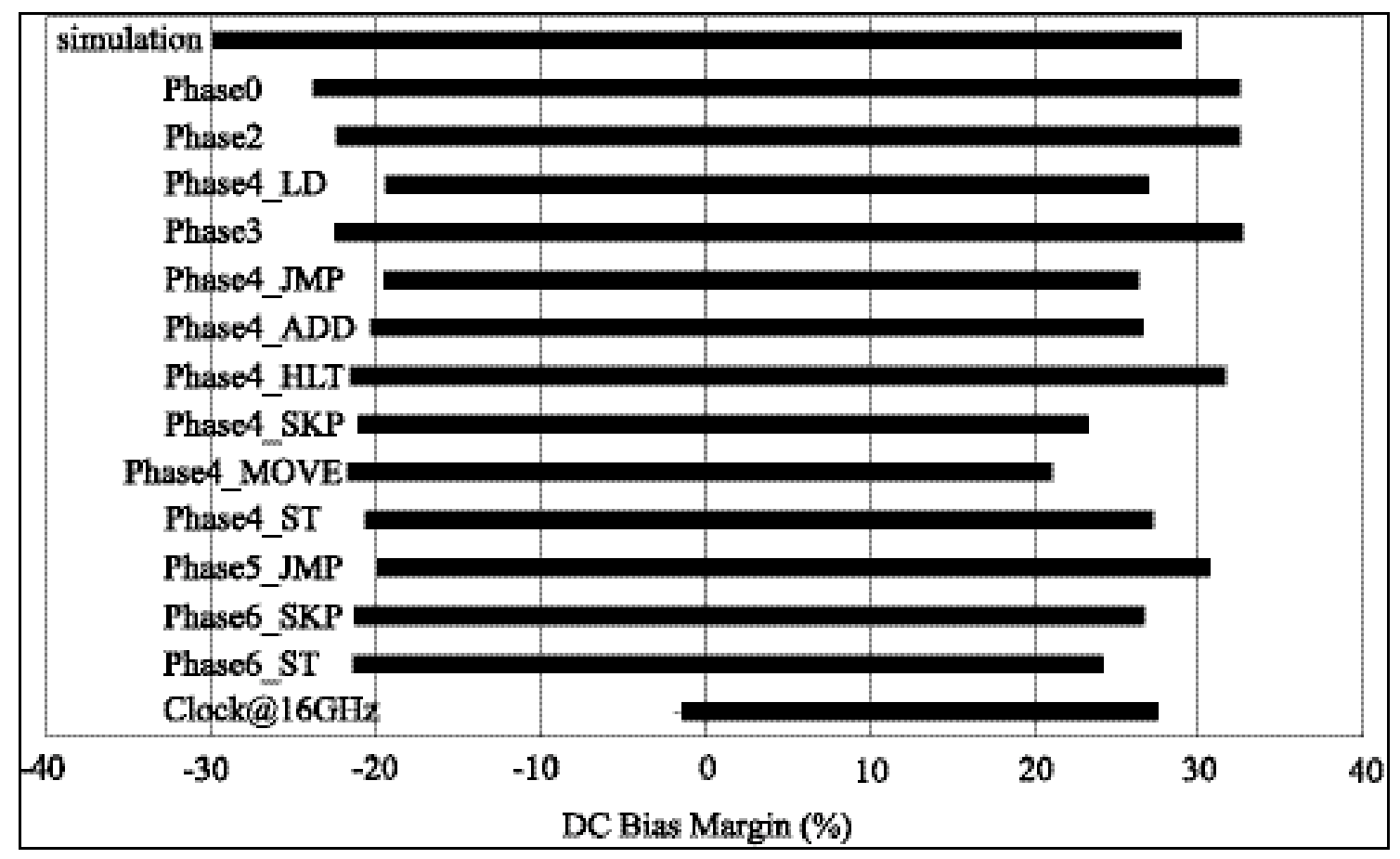

Figure 11 


\section{Table 1}

A sequence table of the controller. Its function is determined by the microprocessor phase and the three-bit opcode defined by $(000)=$ "HLT", $(001)=$ "JMP", $(011)=$ "LD", $(100)=$ "ADD", $(101)=$ "SKP", (110) = "MOVE" and $(111)=$ "ST".

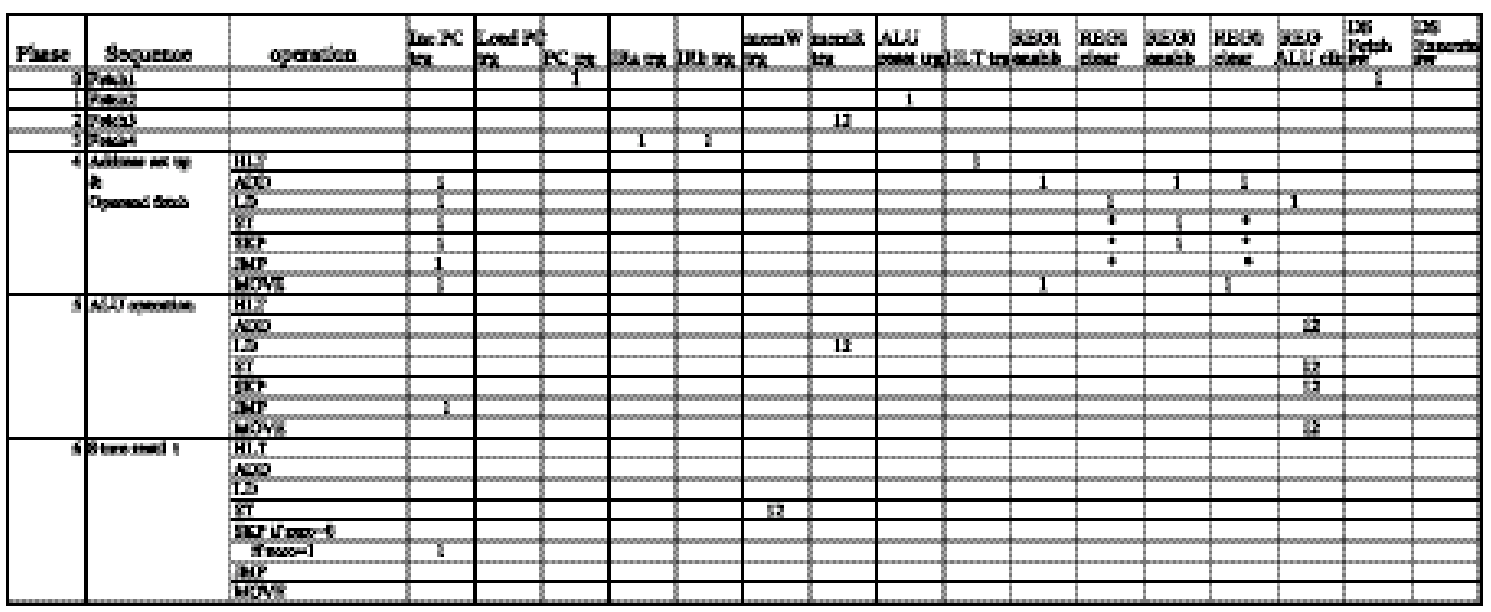

